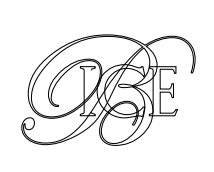

Subdirección General de Estudios y Evaluación de Instrumentos de Política Comercial*

\title{
EL SECTOR EXTERIOR ANTE 2021: RETOS Y OPORTUNIDADES
}

El año 2021 se inicia con perspectivas de recuperación de la actividad económica y comercial internacionales, aunque en un marco de elevada incertidumbre. El sector exterior español, que acusó con intensidad el impacto de la COVID-19 en el año 2020, cuenta con fortalezas, como su amplia base de exportadores regulares, que lo configuran como un factor clave para la recuperación de nuestra economía. Los instrumentos de apoyo a la internacionalización se adaptaron durante 2020 a las necesidades de las empresas frente al impacto de la pandemia. A medio plazo, será preciso un nuevo esfuerzo de adecuación de estas políticas de apoyo para acompañar a las empresas españolas internacionalizadas en el aprovechamiento de sus fortalezas y de las oportunidades que va a ofrecer el escenario internacional poscovid.

Palabras clave: comercio exterior, internacionalización, exportadores regulares, COVID-19, Brexit, política comercial, plan de choque.

Clasificación JEL: F13, F14.

\section{Introducción}

Las perspectivas de recuperación de la actividad económica y comercial internacional para el año 2021 son positivas, a la vista de la mejora de los indicadores económicos en la última parte de 2020, de las expectativas generadas por el desarrollo de las vacunas frente a la COVID-19 y de los planes de estímulo que están poniendo en marcha las principales economías mundiales.

Esta recuperación, aún sujeta a elevados niveles de incertidumbre, tendrá lugar tras un

\footnotetext{
* Secretaria de Estado de Comercio.

Versión de abril de 2021.

DOI: https:/doi.org/10.32796/bice.2021.3134.7184
}

año 2020 marcado por el impacto económico de la pandemia, que se ha reflejado, con intensidad, en el sector exterior.

La crisis sanitaria ha tenido también un elevado impacto en España, cuya economía está muy vinculada al turismo, una de las actividades más afectadas por las medidas de contención adoptadas frente a la COVID-19. Para el año 2021 se espera también una fuerte recuperación de la economía española.

En este artículo se analiza el impacto de la COVID-19 en el sector exterior español y el papel que dicho sector puede desempeñar en la esperada recuperación. Para ello, en el siguiente epígrafe se analiza la situación actual y las perspectivas para el entorno internacional. El epígrafe 3 se centra en la evolución del $\triangleright$ 
Subdirección General de Estudios y Evaluación de Instrumentos de Política Comercial

sector exterior español en 2020, destacando el impacto de la crisis en su estructura sectorial y geográfica. En el epígrafe 4 se estudia el papel desempeñado por los instrumentos de apoyo a la internacionalización para paliar el impacto de la crisis sanitaria y preservar el tejido exportador. Finalmente, el epígrafe 5 se dedica a analizar, desde una perspectiva de medio plazo, las fortalezas que presenta el sector exterior español, su potencial para contribuir al proceso de recuperación económica y el acompañamiento que, para ello, debe recibir de las políticas públicas. El trabajo se cierra con un epígrafe de conclusiones.

\section{El impacto de la COVID-19 en la actividad y el comercio internacionales}

El año 2021 se ha iniciado con perspectivas de recuperación de la actividad económica y el comercio internacionales tras el fuerte impacto de la COVID-19 en 2020. Así, el Fondo Monetario Internacional (FMI) prevé un crecimiento de la economía mundial del $6,0 \%$ en 2021 , frente al descenso del 3,3\% estimado para 2020 (Cuadro 1).

Las previsiones de recuperación de la actividad económica son más optimistas que las que se mantenían hace unos meses, alentadas por unos resultados mejores de lo esperado en el último trimestre del año 2020 , que podrían reflejar una mayor adaptación de las economías a las condiciones impuestas por la pandemia, así como por las esperanzas depositadas en el despliegue de las campañas de vacunación. No obstante, la recuperación continúa sujeta a una elevada incertidumbre y será dispar por sectores, regiones y países, como lo ha sido también el impacto de la pandemia.
CUADRO 1

ESTIMACIÓNY PREVISIONES DEL FMI EN ABRIL DE 2021 (Tasas de variación interanual)

\begin{tabular}{|c|c|c|c|}
\hline & 2020 & 2021 & 2022 \\
\hline Producto mundial & $-3,3$ & 6,0 & 4,4 \\
\hline Economías avanzadas... & $-3,4$ & 5,1 & 3,1 \\
\hline 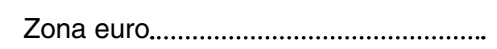 & $-6,6$ & 4,4 & 3,8 \\
\hline 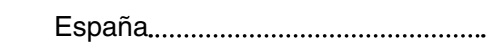 & $-11,0$ & 6,4 & 4,7 \\
\hline Alemania ........................ & $-4,9$ & 3,6 & 3,4 \\
\hline Francia & $-8,2$ & 5,8 & 4,2 \\
\hline Italia............ & $-8,9$ & 4,2 & 3,6 \\
\hline 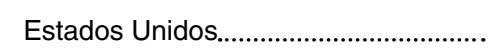 & $-3,5$ & 6,4 & 3,5 \\
\hline 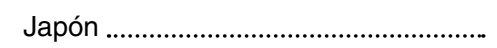 & $-4,8$ & 3,3 & 2,5 \\
\hline Reino Unido............ & $-9,9$ & 5,3 & 5,1 \\
\hline Econ. emergentes y en desarrollo & $-2,2$ & 6,7 & 5,0 \\
\hline Asia $\ldots \ldots \ldots \ldots . . . . . .$. & $-1,0$ & 8,6 & 6,0 \\
\hline China............. & 2,3 & 8,1 & 5,6 \\
\hline 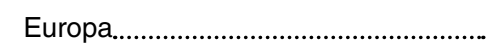 & $-2,0$ & 4,4 & 3,9 \\
\hline América Latina y el Caribe......................... & $-7,0$ & 4,6 & 3,1 \\
\hline Oriente Medio y Asia Central.................. & $-2,9$ & 3,7 & 3,8 \\
\hline África Subsahariana & $-1,9$ & 3,4 & 4,0 \\
\hline Comercio de bienes y servicios (vol.) & $-8,5$ & 8,4 & 6,5 \\
\hline
\end{tabular}

En efecto, los países con estructuras productivas más orientadas a las actividades que se han visto más restringidas por las medidas adoptadas para contener la pandemia y por los cambios voluntarios en los hábitos sociales han registrado descensos más acusados en su actividad. El Gráfico 1 ilustra cómo las economías más orientadas al turismo y los viajes figuran entre las que han registrado mayores caídas del producto interior bruto (PIB).

Para el año 2021, destaca el crecimiento previsto en las economías emergentes de Asia, y en particular en China, frente a los ritmos algo más moderados en las economías avanzadas $\mathrm{y}$, sobre todo, en las emergentes y en desarrollo no asiáticas. Además de las características propias de las distintas $D$ 


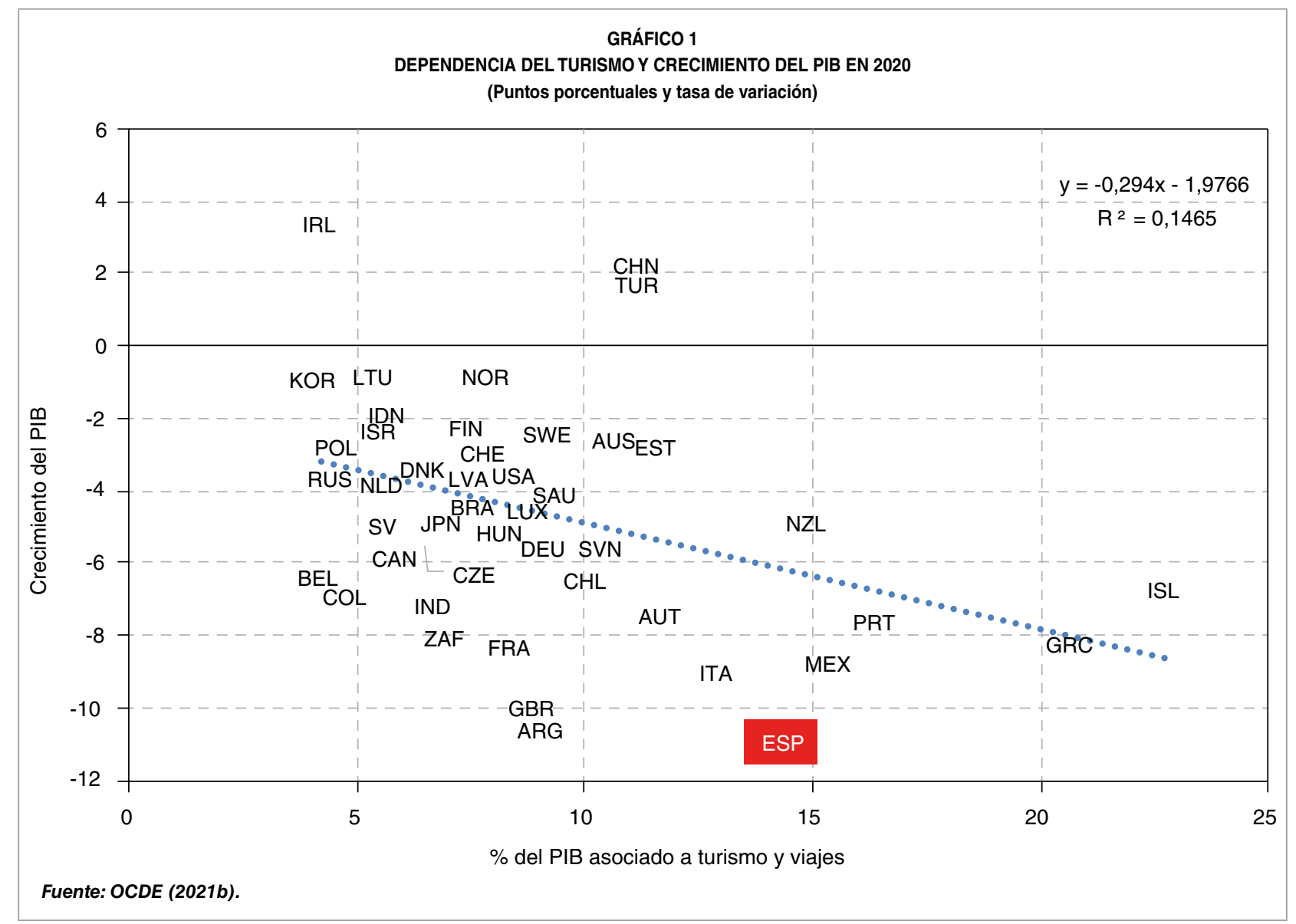

economías y de su grado de exposición a la pandemia y a su impacto económico, el acceso a las vacunas y la capacidad para impulsar planes de recuperación explican las diferencias en las perspectivas nacionales $y$ regionales.

El impacto económico de la pandemia ha afectado con especial intensidad a los intercambios comerciales internacionales. Así, en 2020 el comercio internacional de bienes y servicios registró un descenso del $8,5 \%$, el más acusado desde 2009, año en el que se reflejaron con mayor intensidad los efectos de la Gran Recesión. Las perspectivas para los próximos años son también de recuperación del comercio internacional, con tasas de crecimiento que superarán a las del conjunto de la actividad económica (Gráfico 2).
Este escenario de recuperación de la actividad económica y comercial internacional en los próximos años está sujeto, como se ha señalado, a un elevado nivel de incertidumbre. La progresión del virus, asociada en particular a la capacidad de transmisión de sus mutaciones, y los avances en la vacunación, que requerirán un esfuerzo de cooperación internacional para tener efectos globales, son dos elementos claves para su concreción.

Otro elemento crítico es el alcance y la materialización de los planes de apoyo y reactivación, orientados tanto al sostenimiento de familias y empresas como al impulso a las transiciones ecológica y digital. Entre estos planes pueden destacarse la adopción, por la Unión Europea, del instrumento Next Generation EU, dotado con 750.000 millones de $\triangleright$ 


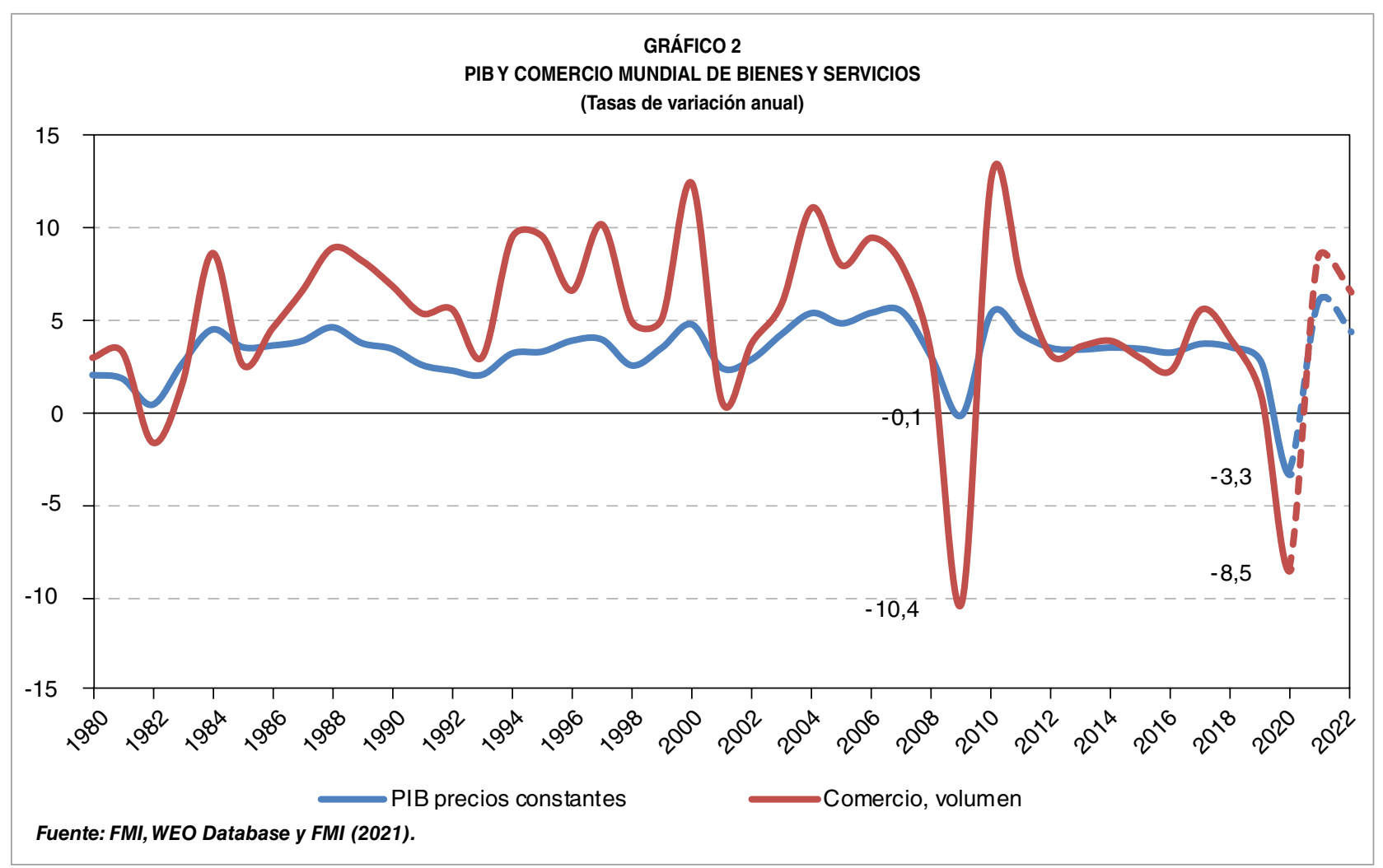

euros (Subdirección General de Asuntos Económicos y Financieros, 2020) o el Plan de Rescate Americano, adoptado en marzo de 2021, con un alcance de 1,9 billones de dólares (OCDE, 2021a).

En gran medida, estos planes de reactivación estarán orientados a facilitar la superación de los retos que la economía global está ya afrontando. Entre estos retos cobran especial importancia los asociados a la digitalización y a la transición ecológica. Los planes de reactivación serán vectores de aceleración de estos cambios y generarán oportunidades de negocio en el marco internacional.

Como puede observarse en el Gráfico 3, el apoyo fiscal que se ha materializado desde el inicio de la crisis sanitaria o que está ya previsto presenta variaciones significativas en distintas economías, lo que contribuirá a que los ritmos de recuperación sean también diferenciados. Así, el apoyo fiscal en EE UU, aproximado por el cambio en la necesidad de financiación neta de las Administraciones públicas, alcanzará el $13,1 \%$ del PIB, frente al $6,8 \%$ en la zona euro o al $4,1 \%$ en Japón.

No obstante, la dimensión de los estímulos fiscales adoptados generará efectos desbordamiento positivos en otras economías. En este sentido, la OCDE estima que el Plan de Rescate Americano podría tener efectos muy significativos en los países más vinculados con Estados Unidos, como Canadá o México, y algo más moderados en otras economías más alejadas, como las de la zona euro o China (Gráfico 3). El impacto será más intenso si los consumidores son más reactivos a las variaciones inmediatas en sus ingresos (el supuesto adoptado en el gráfico) y más moderado si tienen presente el aumento de endeudamiento público asociado a los planes y el previsible incremento de impuestos futuro. También serán especialmente relevantes la velocidad en la $\triangleright$ 


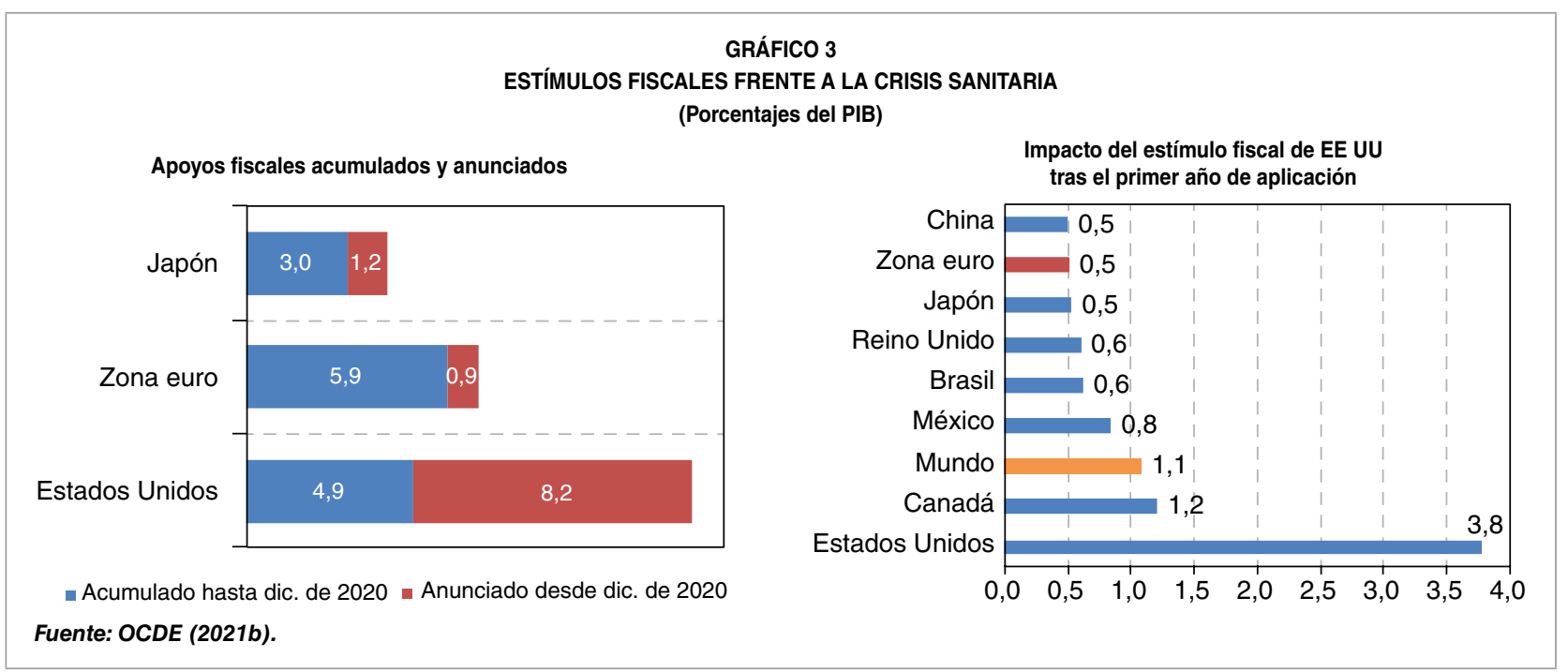

aplicación de las nuevas medidas fiscales anunciadas, su progresivo enfoque hacia los ámbitos en los que se requieran reasignaciones de mano de obra y capital, y la vinculación entre el mantenimiento de los programas de apoyo y la evolución de la crisis sanitaria y de la economía (OCDE, 2021a).

\section{El comercio internacional español en 2020}

En este marco internacional, el sector exterior español ha acusado, durante 2020, los efectos de la crisis sanitaria. Las diferencias entre los ritmos de expansión de la pandemia y de medidas adoptadas para su contención en distintas áreas geográficas, así como los efectos sectoriales asimétricos que han tenido estas medidas, han determinado cambios en la estructura geográfica y sectorial del comercio exterior español.

Así, durante el año 2020 se han registrado reducciones del $20,9 \%$ en los ingresos y del $17,6 \%$ en los pagos asociados al comercio internacional de bienes y servicios (Gráfico 4). Los descensos han sido más intensos en los ingresos y los pagos vinculados al comercio de servicios (con tasas anuales del $-43,9 \%$ y $-30,8 \%$ ) que en los asociados a bienes (con variaciones del $-9,9 \%$, para los ingresos y del $-14,5 \%$, para los pagos).

Un análisis más desagregado permite constatar que esta diferencia se explica por el efecto de las restricciones a la movilidad en los ingresos y pagos por turismo, que han registrado reducciones del $77,3 \%$ y el $69,2 \%$, respectivamente. Los descensos en ingresos y pagos asociados a otros servicios han sido notablemente más moderados, del $9,6 \%$ y $12,2 \%$, respectivamente, e incluso algo menos intensos que los contabilizados para bienes.

El análisis del comercio exterior de bienes revela que, como reflejo del alcance global de la crisis sanitaria, el descenso de las exportaciones españolas según su destino geográfico ha sido generalizado (Cuadro 2). Así, según los datos de comercio declarado, las exportaciones de bienes descendieron un $10,0 \%$ en el conjunto del año 2020. El descenso fue algo más moderado en las exportaciones dirigidas a la Unión Europea (con una tasa de variación anual del $-7,7 \%$ ) y, en particular, a la zona euro $(-7,0 \%)$. Por el contrario, fue más intenso en $\square$ 


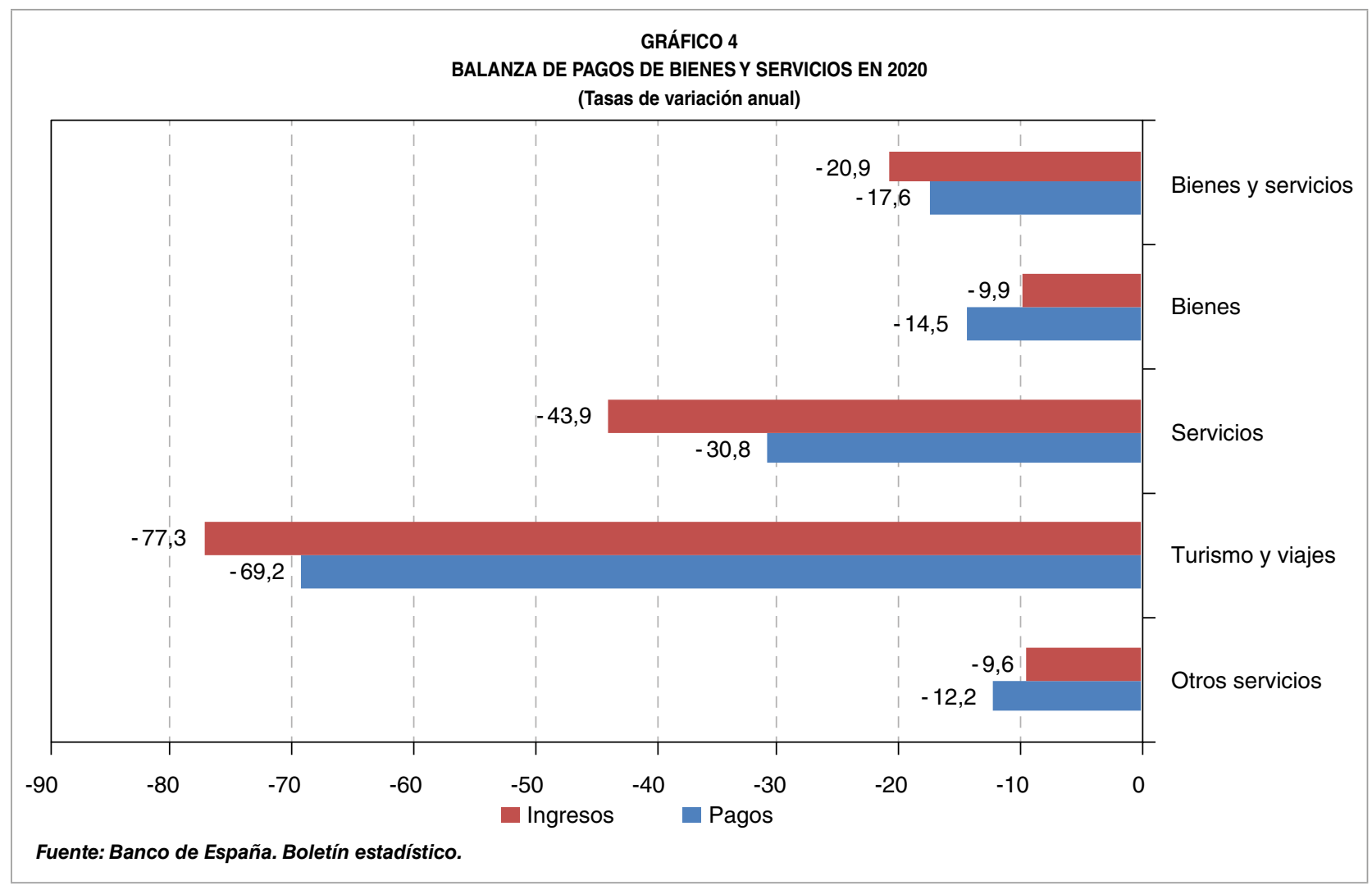

las dirigidas a América Latina, con una caída del $23,8 \%$, y África, con un descenso del $16,3 \%$. Las exportaciones dirigidas a Asia, excluyendo Oriente Medio, se redujeron en un $5,7 \%$. Este descenso, relativamente moderado, se explica por el fuerte crecimiento de las exportaciones a China (con una tasa anual del $20,1 \%$ ), país que tras el esfuerzo de contención de la pandemia logró recuperar un ritmo de crecimiento que ha facilitado el aumento de las ventas españolas a ese destino.

Las importaciones de bienes siguieron, igualmente, una pauta geográfica de descensos generalizados en 2020. En general, estos descensos fueron más intensos que los de las ventas al exterior. Destacaron las reducciones de las compras procedentes de Oriente Medio (con una tasa de variación del $-44,1 \%$ ) y de África (-30,1\%). Como en el caso de las exportaciones, cabe señalar el comportamiento diferencial de las importaciones procedentes de China, que registraron en el conjunto del año un avance del $0,6 \%$.

La evolución sectorial del comercio de bienes refleja también el impacto de la COVID-19. La pandemia ha tenido impactos positivos 0 menos desfavorables en algunos sectores vinculados al consumo de primera necesidad, como la industria de la alimentación o la sanitaria. Por el contrario, los sectores vinculados con la inversión, como los bienes de equipo, o con el consumo duradero, como los vehículos, sufrieron los impactos más severos, en especial en la primera parte del año. El comportamiento de estos sectores refleja tanto la interrupción en las cadenas globales de valor que tuvo lugar al inicio de la crisis, como consecuencia de las medidas de contención adoptadas en diversos países, como del efecto de cambios en los hábitos sociales en las pautas de consumo, así $D$ 
CUADRO 2

COMERCIO EXTERIOR EN 2020: DESGLOSE POR ÁREAS GEOGRÁFICAS

\begin{tabular}{|c|c|c|c|c|c|c|}
\hline \multirow{2}{*}{ Área geográfica } & \multicolumn{3}{|c|}{ Exportaciones } & \multicolumn{3}{|c|}{ Importaciones } \\
\hline & $\%$ total & $\operatorname{tva}(\%)^{\star}$ & contrib. ${ }^{\star \star}$ & $\%$ total & $\operatorname{tva}(\%)^{\star}$ & contrib.** \\
\hline Europa. & 73,0 & $-8,0$ & $-5,7$ & 61,1 & $-13,0$ & $-7,8$ \\
\hline Unión Europea. & 60,5 & $-7,7$ & $-4,5$ & 51,8 & $-11,9$ & $-6,0$ \\
\hline Zona euro..... & 53,2 & $-7,0$ & $-3,6$ & 43,9 & $-12,4$ & $-5,3$ \\
\hline Alemania.......... & 11,3 & $-4,8$ & $-0,5$ & 12,4 & $-14,5$ & $-1,8$ \\
\hline Francia........ & 16,1 & $-3,9$ & $-0,6$ & 10,4 & $-14,6$ & $-1,5$ \\
\hline Italia............... & 7,8 & $-11,8$ & $-0,9$ & 6,5 & $-13,4$ & $-0,9$ \\
\hline 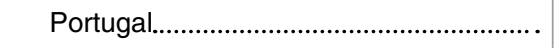 & 7,6 & $-9,7$ & $-0,7$ & 3,9 & $-5,9$ & $-0,2$ \\
\hline Resto UE & 7,3 & $-12,2$ & $-0,9$ & 8,0 & $-8,9$ & $-0,7$ \\
\hline Resto Europa......... & 12,5 & $-9,6$ & $-1,2$ & 9,3 & $-18,6$ & $-1,8$ \\
\hline Reino Unido.................... & 6,5 & $-13,5$ & $-0,9$ & 3,4 & $-20,7$ & $-0,8$ \\
\hline Turquía...... & 1,6 & $-4,6$ & $-0,1$ & 2,3 & $-16,8$ & $-0,4$ \\
\hline América. & 9,9 & $-17,4$ & $-1,9$ & 10,6 & $-14,3$ & $-1,5$ \\
\hline América del Norte. & 5,4 & $-11,5$ & $-0,6$ & 5,6 & $-10,2$ & $-0,5$ \\
\hline 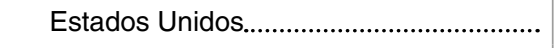 & 4,7 & $-11,2$ & $-0,5$ & 5,1 & $-9,5$ & $-0,5$ \\
\hline América Latina & 4,4 & $-23,8$ & $-1,2$ & 4,7 & $-17,8$ & $-0,9$ \\
\hline Brasil,_._. & 0,9 & $-13,1$ & $-0,1$ & 1,3 & $-7,9$ & $-0,1$ \\
\hline México & 1,2 & $-25,1$ & $-0,4$ & 1,3 & $-25,0$ & $-0,4$ \\
\hline Asia & 9,5 & $-7,1$ & $-0,7$ & 21,1 & $-13,7$ & $-2,8$ \\
\hline 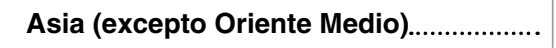 & 7,0 & $-5,7$ & $-0,4$ & 19,3 & $-9,1$ & $-1,7$ \\
\hline China & 3,1 & 20,1 & 0,5 & 10,7 & 0,6 & 0,1 \\
\hline Japón & 1,0 & $-7,6$ & $-0,1$ & 1,1 & $-33,2$ & $-0,4$ \\
\hline Oriente Medio. & 2,6 & $-10,5$ & $-0,3$ & 1,8 & $-44,1$ & $-1,2$ \\
\hline Arabia Saudí.... & 0,7 & $-5,8$ & 0,0 & 0,8 & $-46,1$ & $-0,6$ \\
\hline África.... & 6,0 & $-16,3$ & $-1,0$ & 6,9 & $-30,1$ & $-2,5$ \\
\hline 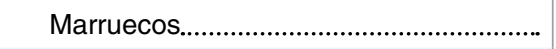 & 2,8 & $-13,3$ & $-0,4$ & 2,3 & $-8,4$ & $-0,2$ \\
\hline Oceanía. & 0,7 & $-4,3$ & 0,0 & 0,2 & $-19,9$ & 0,0 \\
\hline Australia & 0,6 & $-5,7$ & 0,0 & 0,1 & $-32,5$ & 0,0 \\
\hline Otros & 0,9 & $-44,0$ & $-0,6$ & 0,0 & $-52,8$ & 0,0 \\
\hline Total comercio declarado.................................. & 100,0 & $-10,0$ & $-10,0$ & 100,0 & $-14,7$ & $-14,7$ \\
\hline \multicolumn{7}{|c|}{$\begin{array}{l}{ }^{*} \text { A efectos de cálculo de variación interanual, la comparación se hará con los datos provisionales de } 2019 . \\
\text { ** Contribución a la tasa de variación anual de las exportaciones/importaciones totales, en puntos porcentuales. }\end{array}$} \\
\hline $\begin{array}{l}\text { Fuente: S.G. de Estudios y Evaluación de Instrum } \\
\text { tria, Comercio y Turismo, a partir de datos del De }\end{array}$ & $\begin{array}{l}\text { tos de Poli } \\
\text { rtamento a }\end{array}$ & Comercial d & Secretaría d & $\begin{array}{l}\text { tado de C } \\
\text { taria. }\end{array}$ & o del Mir & de Indus- \\
\hline
\end{tabular}

como del aumento de la incertidumbre sobre las decisiones de inversión y consumo. En este sentido, un análisis más desagregado confirma el crecimiento del comercio internacional de algunos bienes esenciales, como alimentos 0 productos sanitarios y medicamentos, así como otros vinculados con el teletrabajo o el ocio en casa (ordenadores, equipos electrónicos o electrodomésticos), mientras que han caído con más intensidad los flujos asociados a la movilidad (productos energéticos y automóvil) o la vida social (productos de la confección).

El sector cuyas exportaciones han evolucionado más favorablemente en 2020 es el de alimentación, bebidas y tabaco (Cuadro 3). Con un peso del $19,6 \%$ de las exportaciones totales, sus ventas al exterior crecieron un $5,5 \% \triangleright$ 
CUADRO 3

COMERCIO EXTERIOR POR SECTORES EN 2020

\begin{tabular}{|c|c|c|c|c|c|c|}
\hline \multirow{2}{*}{ Sector } & \multicolumn{3}{|c|}{ Exportaciones } & \multicolumn{3}{|c|}{ Importaciones } \\
\hline & $\%$ total & tva (\%) & contrib. & $\%$ total & tva (\%) & contrib. \\
\hline Alimentación, bebidas y tabaco............. & 19,6 & 5,5 & 0,9 & 12,4 & $-4,8$ & $-0,5$ \\
\hline Productos energéticos & 4,8 & $-40,9$ & $-3,0$ & 9,8 & $-39,1$ & $-5,4$ \\
\hline Materias primas & 2,3 & $-14,4$ & $-0,4$ & 3,1 & $-16,6$ & $-0,5$ \\
\hline Semimanufacturas no químicas............. & 10,1 & $-10,4$ & $-1,1$ & 7,0 & $-16,7$ & $-1,2$ \\
\hline Productos químicos & 15,5 & $-3,6$ & $-0,5$ & 18,0 & $-3,2$ & $-0,5$ \\
\hline 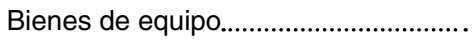 & 19,8 & $-12,6$ & $-2,6$ & 22,6 & $-9,7$ & $-2,1$ \\
\hline 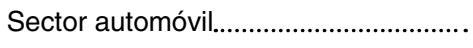 & 14,8 & $-12,9$ & $-2,0$ & 11,1 & $-24,7$ & $-3,1$ \\
\hline Bienes de consumo duradero.................. & 1,7 & $-2,7$ & 0,0 & 2,9 & $-5,0$ & $-0,1$ \\
\hline Manufacturas de consumo........................... & 9,5 & $-15,1$ & $-1,5$ & 12,6 & $-10,6$ & $-1,3$ \\
\hline Otras mercancías......... & 1,8 & 9,2 & 0,1 & 0,5 & $-6,6$ & 0,0 \\
\hline Total comercio declarado & 100,0 & $-10,0$ & $-10,0$ & 100,0 & $-14,7$ & $-14,7$ \\
\hline
\end{tabular}

respecto a 2019. Dentro del sector destacaron por su evolución positiva las exportaciones de productos cárnicos, con un aumento del $16,2 \%$, y las de frutas, hortalizas y legumbres, con un crecimiento del $6,0 \%$.

Entre las caídas, destaca de forma especial el descenso de las exportaciones de productos energéticos $(4,8 \%$ de las exportaciones totales, $-40,9 \%$ interanual) que ha sido paralelo a la disminución de las importaciones y que responde a una combinación de menores volúmenes y caída de precios internacionales (con un descenso del 39,1\%). También han caído las exportaciones del sector automóvil $(14,8 \%$ del total, $-12,9 \%$ interanual) y bienes de equipo (19,8\% del total, $-12,6 \%$ interanual). En estos dos casos, tras una intensa caída al inicio de la pandemia, las exportaciones se han recuperado en los últimos meses, alcanzando de nuevo tasas positivas. Destaca la recuperación de las exportaciones de automóviles, con crecimientos interanuales durante toda la segunda mitad de 2020.

En la vertiente importadora, los descensos han sido generalizados, con una caída más intensa de los productos energéticos, como ya se ha señalado. Los descensos más moderados se han registrado en sectores como productos químicos $(-3,2 \%)$ y alimentación, bebidas y tabaco $(-4,8 \%)$. En ambos casos se trata de sectores que incluyen productos, como los farmacéuticos o alimentarios, relativamente beneficiados por la crisis sanitaria.

Si se desciende a nivel de subsectores (Gráfico 5), las mayores contribuciones positivas a la tasa de variación anual de las exportaciones $(-10,0 \%)$ han sido las de productos cárnicos $(0,5$ puntos porcentuales o $\mathrm{pp})$ y frutas, hortalizas y legumbres (0,4 pp); y por el lado negativo, petróleo y derivados $(-2,9 \mathrm{pp})$ y automóviles y motos (-1,0 pp). En el ámbito de las importaciones, destacan por su contribución positiva los medicamentos (0,3 pp) y aceites y grasas $(0,2 p p)$; y por su contribución negativa, petróleo y derivados $(-4,4 \mathrm{pp})$, automóviles y motos (-2,0 pp) y componentes del automóvil (-1,1 pp).

Finalmente, conviene señalar que el perfil mensual de la evolución del comercio de bienes durante el año 2020 revela una tendencia a la recuperación desde el mes de junio (Gráfico 6). Así, el impacto repentino de la $\triangleright$ 


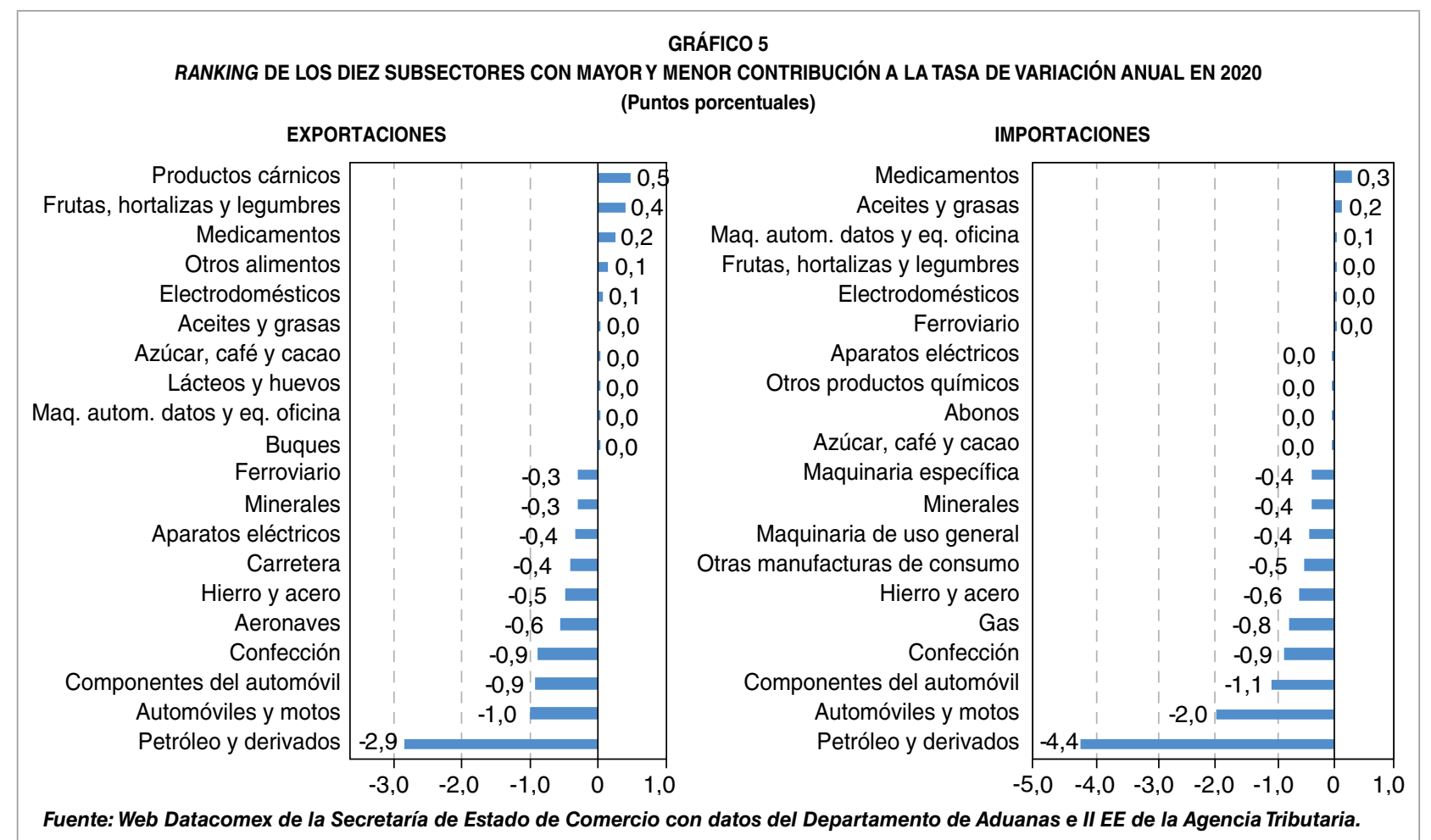

pandemia provocó un colapso del comercio exterior de bienes a partir de mediados de marzo, que alcanzó su punto más bajo en abril y mayo, con caídas de las exportaciones cercanas al $40 \%$ interanual, coincidiendo con la primera ola de la COVID-19 y la necesidad de adoptar severas medidas de restricción de la movilidad en España y en la mayoría de nuestros principales socios comerciales. Desde junio se viene produciendo una recuperación progresiva de los flujos de comercio que han permitido que las exportaciones de bienes, en diciembre de 2020 , superaran ligeramente $(0,9 \%)$ a las del mismo mes de 2019.

Esta línea de recuperación se interrumpió en enero de 2021, mes en el que se registraron descensos interanuales tanto de las exportaciones $(-11,4 \%)$ como de las importaciones $(-16,4 \%)$. En febrero se registraron también descensos interanuales, aunque más moderados, del $1,9 \%$ en las exportaciones y del $5,7 \%$ en las importaciones. Conviene tener presente que algunos factores coyunturales, como la efectiva entrada en vigor de las nuevas condiciones comerciales con Reino Unido o el impacto de la tormenta Filomena han contribuido a esta evolución en los primeros meses de 2021, por lo que las perspectivas para el conjunto del año pueden considerarse más favorables.

\section{El plan de choque frente a la COVID-19}

El impacto de la crisis sanitaria internacional ha sido particularmente severo en España dado el peso que tienen en nuestra economía actividades que, como las relacionadas con el turismo, se han visto especialmente afectadas por las medidas adoptadas para la contención de la pandemia. El comercio exterior español, como se ha expuesto en el epígrafe anterior, ha acusado también con intensidad el impacto de la crisis. 


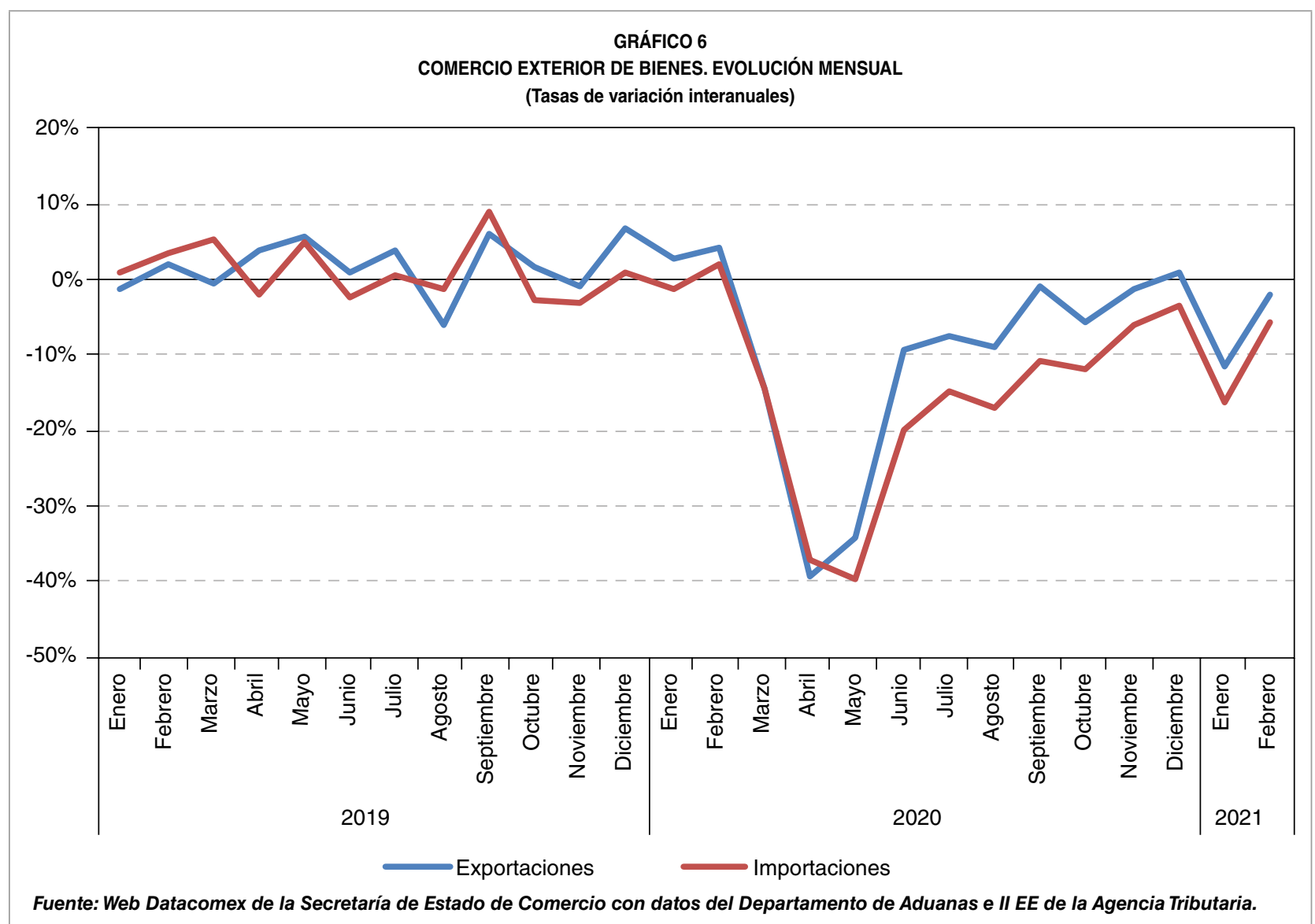

En este contexto, la estrategia de política económica desplegada para hacer frente al impacto de la COVID-19 ha tenido, como uno de sus ejes principales, salvaguardar el tejido empresarial (Dirección General de Política Económica, 2020). Este objetivo cobra especial relevancia ante la percepción de que las medidas de contención tienen un horizonte temporal y que su levantamiento permitirá una rápida recuperación, si el tejido empresarial está preparado para afrontarla.

Con este fin se han puesto diversos instrumentos a disposición de las empresas. Entre ellos destacan las líneas de avales públicos para facilitar la financiación de las empresas, por un importe global de 100.000 millones de euros, y la flexibilización de condiciones de los Expedientes de Regulación Temporal de Empleo, a los que, en el punto más alto registrado durante la pandemia, en abril de 2020, llegaron a acogerse más de 3,6 millones de trabajadores.

Las empresas internacionalizadas han hecho uso de estas medidas de carácter general, que persiguen facilitar la financiación de las empresas y aliviar sus costes, al tiempo que mantienen el vínculo con sus trabajadores. De esta forma, se asegura el mantenimiento de las empresas y los puestos de trabajo, y se facilita, como se ha señalado, la ulterior recuperación económica.

No obstante, el proceso de internacionalización de las empresas, en particular de las pymes, requiere apoyos específicos, que han tenido que adaptarse al complejo escenario internacional de 2020. El Plan de choque frente a la COVID-19 en apoyo a la internacionalización (Secretaría de Estado de Comercio, 2020) $D$ 
(FIEM) ha ampliado los fondos disponibles, flexibilizado las condiciones de acceso y reforzado la línea FIEM-PYME, incrementada en 100 millones de euros. CESCE ha puesto en marcha una línea de cobertura del riesgo de 2.000 millones de euros destinada a asegurar la financiación bancaria de circulante a empresas exportadoras afectadas por la COVID-19. También ha cubierto, por cuenta del Estado, operaciones de exportación a corto plazo en países OCDE y ha ampliado y adaptado la línea especial de fianzas para pymes y empresas no cotizadas en el proceso de internacionalización, cuya dotación actual asciende a 500 millones de euros, con el fin de que las empresas puedan emitir avales técnicos y optar a contratos. COFIDES ha aplicado el mecanismo fast track de aplazamientos de cuotas de deudas de empresas afectadas por la crisis, que se aplica tanto a las operaciones de COFIDES como a las del Fondo para Inversiones en el Exterior (FIEX) y el Fondo para Operaciones de Inversión en el Exterior de la pyme (FONPYME). Se han facilitado, además, aplazamientos de pagos de operaciones y devoluciones de cuotas por actividades canceladas 0 en las que las empresas no pueden participar, con el objetivo de contribuir al alivio financiero de las empresas.

- Refuerzo de la imagen de España. La estrategia de refuerzo de la imagen de España adquirió especial importancia en los momentos en que la evolución de la pandemia amenazaba con deteriorarla. En particular, cabe recordar la especial incidencia en España de su primera ola, frente a un desarrollo más suave en otras economías y a una rápida contención en algunos países asiáticos. Así, para promover una imagen asociada a la competitividad y prevenir un eventual deterioro de la misma ante el impacto de la COVID-19, ICEX ha lanzado nuevas estrategias de comunicación e imagen. Además, se han reforzado los instrumentos de comunicación en destino de las Oficinas Económicas y Comerciales (redes sociales y perfiles). También ICEX Invest in Spain ha potenciado su estrategia aftercare, con el objetivo de retener la inversión de empresas extranjeras en España, en colaboración con la Direcciones Territoriales y Provinciales de Comercio y con las agencias de atracción de inversiones de las comunidades autónomas.

- Aprovechamiento de los acuerdos comerciales de la UE. Se ha impulsado la apertura de los mercados, influyendo en la UE y en foros multilaterales como la OMC, la OCDE, la UNCTAD o el G20, para contrarrestar las presiones proteccionistas alentadas por la crisis sanitaria. Además, se han intensificado los esfuerzos para facilitar a las empresas un mejor aprovechamiento de las oportunidades que ofrecen los acuerdos comerciales de la UE.

Estas líneas de actuación responden al objetivo de salvaguardar el tejido de empresas internacionalizadas ante el impacto de la crisis sanitaria que se percibe como un shock adverso, pero de carácter temporal. Esta percepción es, además, consistente con la que han mantenido las propias empresas exportadoras.

Así, en la Encuesta de Coyuntura de la Exportación de la Secretaría de Estado de Comercio del segundo trimestre de 2020 se incluyó $\square$ 
un conjunto de preguntas relativas al impacto de la COVID-19. De acuerdo con esta encuesta, el $73 \%$ de las empresas que exportan regularmente esperaba recuperar su volumen de exportaciones previo a la crisis antes de doce meses, mientras que solo un $5 \%$ espera tardar más de veinticuatro meses en hacerlo.

Las líneas de acción del plan de choque también responden, en términos generales, a las necesidades percibidas por las empresas que exportan regularmente. Así, de acuerdo con la encuesta citada, las empresas consideraban prioritario reforzar la cobertura de riesgos asociados al comercio exterior (seguro de crédito exportación, inversión, avales), las campañas para mejorar la imagen internacional de España, el acceso a contactos de potenciales clientes o socios locales en el mercado de destino y el apoyo logístico.

\section{Fortalezas y retos a medio plazo}

En el año 2021, como se ha señalado, se espera una recuperación de la actividad y el comercio internacionales. El FMI prevé que el PIB mundial crezca un 6,0\% y el comercio de bienes y servicios un $8,4 \%$ (FMI, 2021). La Organización Mundial de Comercio (OMC) prevé que el comercio mundial de mercancías aumente en un $8,0 \%$ en 2021 , tras haber disminuido un 5,3\% en 2020 (OMC, 2021).

El sector exterior español, a pesar del notable impacto de la crisis sanitaria durante 2020, se encuentra en una buena posición para aprovechar la recuperación del comercio internacional en 2021. Las políticas de apoyo a la internacionalización, que mostraron una notable capacidad de reacción a corto plazo durante 2020, deberán realizar un nuevo esfuerzo de adaptación a la realidad del año 2021, facilitando la transición de las empresas a un escenario poscovid que, previsiblemente, presentará algunas diferencias con relación al existente antes de la pandemia.

En efecto, desde la Gran Recesión de 2008 el sector exterior español ha registrado una tendencia positiva y ha acumulado algunas fortalezas que lo sitúan en una buena posición para aprovechar la recuperación de la actividad y el comercio internacionales en 2021. El lector interesado puede acceder a un análisis más detallado de dichas tendencias y fortalezas en Subdirección General de Estudios y Evaluación de Instrumentos de Política Comercial (2020). A continuación, se resumen sus principales rasgos y se analiza su evolución durante 2020.

La primera de estas fortalezas es la ampliación de la base exportadora, que ha aumentado de forma ininterrumpida, contando con más de 55.000 empresas que exportan de manera regular. Hay que destacar que, durante 2020, el número de exportadores regulares ha continuado aumentando, con un crecimiento anual del 4,1\% (Gráfico 7). Esta consolidación del tejido exportador, incluso en las difíciles circunstancias del año 2020, favorecida por las medidas adoptadas en el plan de choque, es una sólida base para que el sector exterior pueda contribuir a la recuperación económica.

Un segundo rasgo positivo es la diversificación geográfica de la exportación, con una menor dependencia de la zona euro (a la que se dirigen en 2020 un 53,2\% del total) y un mayor peso de las exportaciones a Asia (9,5\%), a África $(6,0 \%)$ y a América $(9,9 \%)$, donde gana peso Norteamérica frente a América Latina. En el año 2020 esta tendencia se ha moderado en algunos aspectos. Así, las exportaciones a la zona euro han recuperado algo de peso sobre el total (con un aumento de 1,7 pp), en $\triangleright$ 
detrimento de las dirigidas a América (cuyo peso en el total descendió 0,9 pp). Hay que destacar que las ventas a América Latina se han visto especialmente afectadas por la crisis. Las exportaciones a Asia, por el contrario, han continuado ganando peso sobre el total (con un crecimiento de $0,3 \mathrm{pp}$ ), impulsadas por el buen comportamiento de las ventas a China. En todo caso, en una perspectiva de medio plazo, la mayor diversificación geográfica de la exportación española favorecerá el aprovechamiento por parte de las empresas españolas de las oportunidades que ofrezcan los mercados exteriores de distintas áreas geográficas.

Una tercera fortaleza es el creciente peso del comercio exterior de servicios, en particular de los servicios no turísticos, cuyas exportaciones se cuadriplicaron entre los años 2000 y 2019. Las exportaciones de servicios no turísticos, como se ha analizado en el epígrafe 2, se han visto también afectadas por la pandemia durante 2020. No obstante, su descenso ha sido más moderado que el de las exportaciones de bienes y cabe esperar que, en el medio plazo, mantengan el elevado potencial de crecimiento que ha caracterizado su evolución en los años anteriores.

Finalmente, hay que destacar el grado de inserción de España en las cadenas globales de valor (CGV), algo superior al promedio de los países de la UE y la OCDE. Esta favorable inserción en las CGV, muy vinculadas al comercio y la inversión internacionales, revela la capacidad de adaptación de las empresas españolas ante la reorganización en los últimos años de la producción mundial. Las perspectivas a medio plazo apuntan a una posible reestructuración de las CGV, buscando una mayor resiliencia y adquiriendo una dimensión más regional (véase Díaz-Mora et al., 2020 para una aplicación al caso de la península ibérica). Las empresas españolas deberán, por lo $D$

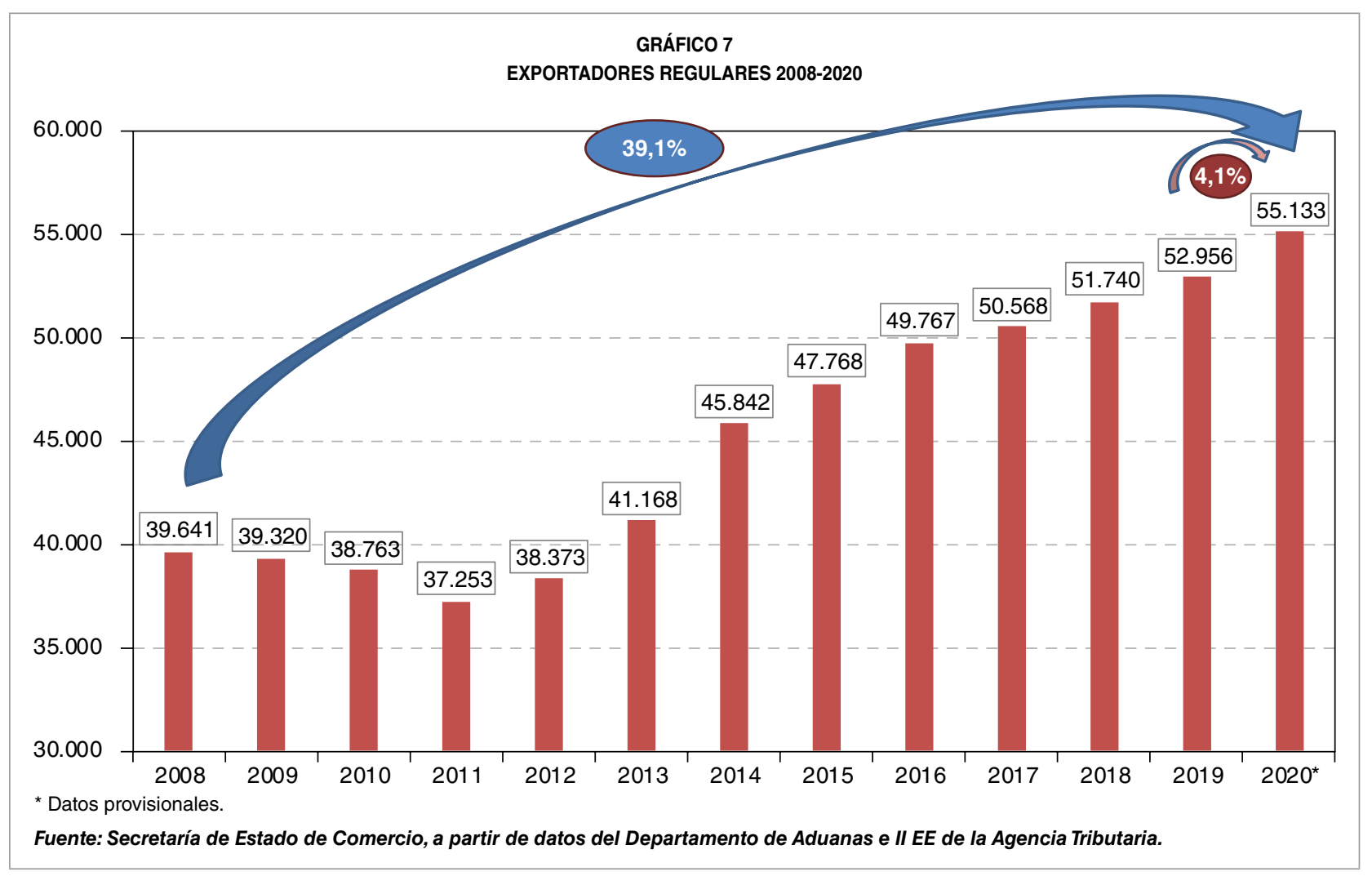


tanto, reaccionar ante este proceso y aprovechar, con el apoyo de las políticas públicas, las oportunidades que puedan presentarse.

Para aprovechar este escenario, en el que se espera una recuperación internacional, es necesario que las políticas de apoyo a la internacionalización se reconfiguren, adaptándose a las condiciones previsibles y facilitando que las empresas españolas aprovechen sus fortalezas. Será preciso mantener las actuaciones del plan de choque mientras se sigan presentando las dificultades de acceso a la financiación y a los mercados que las han motivado, pero además es necesario que los instrumentos de apoyo a la internacionalización faciliten la transformación del sector exterior español y su adaptación a las nuevas tendencias, potenciando su competitividad y su resiliencia frente a posibles futuros shocks.

En este sentido, las previsiones apuntan a que la recuperación de la actividad económica será desigual, por países y por sectores. Aunque estas previsiones continúan sujetas a un elevado nivel de incertidumbre, también sugieren que algunos de los cambios inducidos o acelerados por la COVID-19 tendrán un carácter estructural, afectando también a la esfera internacional. Entre ellos, puede citarse la aceleración de los procesos de digitalización y transformación ecológica, que supondrán tanto un reto como una fuente de oportunidades para las empresas internacionalizadas, la reconfiguración de las cadenas globales de valor o el posible resurgimiento de tensiones comerciales.

Como se ha señalado, los planes de reactivación que están desplegando las principales economías internacionales pueden acelerar algunos de estos cambios, en particular los asociados con la transformación ecológica y digital, y generar así, además, oportunidades de negocio para las empresas españolas internacionalizadas con capacidades en estos ámbitos.

Frente a estas tendencias y estos cambios, el próximo Plan de Acción para la Internacionalización de la Economía Española 20212022, de inminente aprobación en el momento de redactar este artículo, deberá adoptar las acciones necesarias para conseguir que el sector exterior se configure en los próximos años como un pilar de crecimiento económico y para aumentar su resiliencia frente a eventuales shocks. Entre estas acciones será preciso incluir:

- Una revisión de las prioridades geográfico-sectoriales del apoyo a la internacionalización, articuladas en torno a la estrategia de Países con Acción Sectorial Estratégica (PASE), para adaptarlas a las nuevas tendencias internacionales.

- Medidas para continuar progresando en la adecuación de los instrumentos de apoyo a las necesidades concretas de cada tipo de empresa, con especial atención a las pymes, para maximizar su eficacia y dotarlas de mayor fortaleza.

- Acciones para acompañar a las empresas internacionalizadas en su proceso de digitalización, así como en la adopción de estrategias de sostenibilidad, que se configura como una variable de creciente importancia para su competitividad.

- Una estrategia de refuerzo de la seguridad de las cadenas globales de valor, impulsando un marco estable basado en reglas, que permita diversificar las fuentes de abastecimiento y crear marcos cooperativos para el suministro de productos estratégicos, en línea con lo que plantea la Comunicación de la $\triangleright$ 
Comisión Europea sobre revisión de la política comercial, por una política comercial abierta, sostenible y firme (Comisión Europea, 2021).

- Medidas que faciliten que las empresas españolas aprovechen las oportunidades que se van a generar en los mercados internacionales como consecuencia de la aceleración del proceso de digitalización, de transición ecológica y lucha contra el cambio climático y del crecimiento del comercio de servicios.

\section{Conclusiones}

El año 2021 se inicia con perspectivas de recuperación de la actividad y el comercio internacionales, tras el fuerte impacto de la COVID-19 en 2020. Esta recuperación está sujeta aún a una elevada incertidumbre derivada, entre otras variables, de la propia evolución de la pandemia, el éxito de las campañas de vacunación y la capacidad para aplicar políticas públicas de estímulo económico.

España no ha sido ajena al impacto de la pandemia en 2020. En particular, su sector exterior acusó con intensidad la desaceleración del comercio internacional y, muy especialmente, la caída del comercio de servicios turísticos. Sin embargo, desde el mes de junio se ha observado una tendencia de recuperación que podría consolidarse en 2021.

El impulso del sector exterior es clave para la recuperación de la actividad económica. Como ya ocurrió tras la Gran Recesión de 2008, el dinamismo del sector exterior es necesario para alcanzar un crecimiento económico sostenible. El sector exterior acumula fortalezas, entre las que destaca la ampliación de la base de empresas exportadoras, que pueden facilitar una rápida recuperación de los ritmos de crecimiento.

Las políticas de apoyo a la internacionalización se han adaptado con prontitud al impacto de la COVID-19, contribuyendo, a través del plan de choque adoptado en 2020, a preservar esta base de empresas exportadoras.

A medio plazo, es necesario otro esfuerzo de adecuación, a través del Plan de Acción para la Internacionalización de la Economía Española 2021-2022, que propicie que las políticas de apoyo acompañen a las empresas internacionalizadas en su esfuerzo de adaptación a los nuevos parámetros que definirán el escenario internacional poscovid y aprovechen las oportunidades que se van a generar en este escenario.

\section{Bibliografía}

Díaz-Mora, C., Gandoy Juste, R., y González-Díaz, B. (2020). El momento de las cadenas regionales de valor: la integración comercial en la Península Ibérica. Cuadernos Económicos de ICE, (100). https://doi.org/10.32796/cice.2020.100.7116

Dirección General de Política Económica (2020). Política económica ante la emergencia del coronavirus. Boletín Económico de ICE, (3122). https://doi.org/10.32796/bice.2020.3122.6997

Fondo Monetario Internacional (2021). Perspectivas de la economía mundial, abril de 2021. https://www. imf.org/en/Publications/WEO/Issues/2021/03/23/ world-economic-outlook-april-2021

Organización de Cooperación y Desarrollo Económico (2021a). OECD Economic Outlook, Interim Report March 2021. OECD Publishing. París. https://doi.org/10.1787/34bfd999-en

Organización de Cooperación y Desarrollo Económico (2021b). Selected figures and data from the OECD Interim Economic Oulook presentation, March 2021. https://www.oecd.org/ perspectivas-economicas/\#presentation $\quad \triangleright$ 
Organización Mundial de Comercio (31 de marzo de 2021). Estadísticas y perspectivas comerciales. El comercio mundial listo para una recuperación sólida, aunque desigual, después de la pandemia de COVID-19 (Comunicado de prensa, PRESS/876). https://www.wto.org/spanish/ news_s/pres21_s/pr876_s.pdf

Secretaría de Estado de Comercio (2020). Plan de choque frente a la COVID-19 en apoyo a la internacionalización. https://comercio.gob.es/es-es/ estrategia_internacionalizacion/Paginas/ plan-choque-frente-COVID-19-apoyo-internacionalizacion.aspx

Subdirección General de Asuntos Económicos y Financieros (2020). Marco Financiero Plurianual 2021-2027 y Plan de Recuperación para Europa
pos-COVID-19. Boletín Económico de ICE, (3127). https://doi.org/10.32796/bice.2020.3127.7086

Subdirección General de Estudios y Evaluación de Instrumentos de Política Comercial (2020). El comercio exterior ante los retos de 2020. Boletín Económico de ICE, (3121). https://doi.org/ 10.32796/bice.2020.3121.6983

Unión Europea (2021).Communication (COMM(2021) 66 final) from the Commission to the European Parliament, the Council, the European and Economic and Social Committee of the Regions, Trade Policy Review. An Open, Sustainable and Assertive Trade Policy. Diario Oficial de la Unión Europea. https://trade.ec.europa.eu/doclib/docs/2021/february/tradoc_159438.pdf 
